\title{
A hollow cathode arc as a high intensity beam source for ground state and metastable noble gas atoms in the $\mathrm{eV}$ translational energy range
}

\section{Citation for published version (APA):}

Theuws, P. G. A., Beijerinck, H. C. W., Verster, N. F., \& Schram, D. C. (1982). A hollow cathode arc as a high intensity beam source for ground state and metastable noble gas atoms in the $\mathrm{eV}$ translational energy range. Journal of Physics E: Scientific Instruments, 15(5), 573-579. https://doi.org/10.1088/0022-3735/15/5/023

DOI:

10.1088/0022-3735/15/5/023

Document status and date:

Published: 01/01/1982

Document Version:

Publisher's PDF, also known as Version of Record (includes final page, issue and volume numbers)

\section{Please check the document version of this publication:}

- A submitted manuscript is the version of the article upon submission and before peer-review. There can be important differences between the submitted version and the official published version of record. People interested in the research are advised to contact the author for the final version of the publication, or visit the DOI to the publisher's website.

- The final author version and the galley proof are versions of the publication after peer review.

- The final published version features the final layout of the paper including the volume, issue and page numbers.

Link to publication

\footnotetext{
General rights

- You may freely distribute the URL identifying the publication in the public portal. follow below link for the End User Agreement:

www.tue.nl/taverne

\section{Take down policy}

If you believe that this document breaches copyright please contact us at:

openaccess@tue.nl

providing details and we will investigate your claim.
}

Copyright and moral rights for the publications made accessible in the public portal are retained by the authors and/or other copyright owners and it is a condition of accessing publications that users recognise and abide by the legal requirements associated with these rights.

- Users may download and print one copy of any publication from the public portal for the purpose of private study or research.

- You may not further distribute the material or use it for any profit-making activity or commercial gain

If the publication is distributed under the terms of Article 25fa of the Dutch Copyright Act, indicated by the "Taverne" license above, please 


\section{A hollow cathode arc as a high intensity beam source for ground state and metastable noble gas atoms in the $\mathrm{eV}$ translational energy range}

\section{P G A Theuws, H C W Beijerinck, N F Verster and D C Schram}

Physics Department, Eindhoven University of Technology, Eindhoven, The Netherlands

Received 21 October 1981, in final form 27 November 1981

Abstract. The medium density plasma column $\left(n \approx 10^{22} \mathrm{~m}^{-3}\right)$ close to the exit of the cathode of a hollow cathode arc is highly ionised, with ion and electron temperatures $T_{1} \approx 0.5-1.5 \mathrm{eV}$ and $T_{\mathrm{e}} \approx 3 \mathrm{eV}$, respectively. By sampling this plasma with an orifice in the end anode (at a floating potential) a high intensity beam with a broad velocity distribution is obtained for the noble gas atoms $\mathrm{Ne}, \mathrm{Ar}$ and $\mathrm{Kr}$, both in the ground state and in metastable states $n^{*}$. Typical centre-line intensities for argon are $I(0)=2 \times 10^{18} \mathrm{~s}^{-1} \mathrm{sr}^{-1}, T=2 \times 10^{4} \mathrm{~K}$ and $I(0)_{n^{*}}=2 \times 10^{14} \mathrm{~s}^{-1} \mathrm{sr}^{-1}, T_{n^{*}}=3 \times 10^{4} \mathrm{~K}$. These values are in good agreement with model calculations of the process of molecular beam sampling, which are discussed in this paper. The source is of a simple design and easy to operate. The life time of the tungsten (or tantalum) hollow cathode is typically $40 \mathrm{~h}$ for argon and krypton. The shortest life time is for neon with a tungsten cathode, due to the high operating temperature $T \approx 3000 \mathrm{~K}$ of the cathode tip.

\section{Introduction}

In the past much effort has been put in to the development of molecular beam sources of ground state atoms and metastable atoms in the super-thermal energy range $(0.3<E<10 \mathrm{eV})$ (Young et al 1969, Way et al 1976, Bickes et al 1976, Anderson et al 1965). For the production of fast ground state atoms and metastable atoms the method of resonant and near resonant charge exchange of ions with the parent ground state atom (fast ground state atoms) or the corresponding alkali atoms (fast metastable atoms) is very efficient for energies $E>10 \mathrm{eV}$ (Morgenstern et al 1973, Gillen et al 1976). However, for lower energies the intensity of the molecular beam is limited due to space charge effects of the ion beam (Anderson et al 1965). The method of seeded beams, i.e. a supersonic expansion of a trace of the desired (heavy) ground state atom in a light carrier gas like $\mathrm{He}$ or $\mathrm{H}_{2}$, results in high intensities (Anderson 1974) with narrow velocity distributions in the desired energy range. Fast metastable atoms can be produced by electron excitation of a seeded beam. However, great care has to be taken that no traces of metastable carrier gas atoms remain in the beam. Moreover, for accurate measurements of the velocity dependency of total cross sections for elastic or inelastic scattering the combination of a broad velocity distribution with a time-of-flight method for velocity analysis can be rather favourable (Van der Kam 1981, Van der Kam et al 1981). For differential cross section measurements, which in general are performed at only a single velocity, the broad velocity distribution is rather disadvantageous.

Our method is based on the production of a molecular beam from an approximately 50\% ionised plasma with densities at such a value that the ion temperature $T_{\mathrm{i}}$ approaches the electron temperature $T_{e}$. With $T_{e}$ in the order of $3-10 \mathrm{eV}$ the ion temperature will vary from 0.2 to a few $\mathrm{eV}$. Fast ground state atoms are produced in collisions between ions and atoms, i.e. charge exchange and elastic collisions. This results in ground state atoms in the desired velocity range. Because the intensity of the resulting beam depends on the product of the neutral and ion density, we expect optimum results for a highly $(\approx 50 \%)$ ionised plasma. Fast metastable atoms are produced by collisions between electrons and fast ground state atoms (excitation) and between ions and slow metastable atoms with charge exchange. Maximum intensities are expected for high $T_{\mathrm{e}}$ and a high neutral density.

As these plasma conditions are met in a magnetically stabilised hollow cathode arc (HCA), we have chosen to use this type of discharge.

\section{Theory}

A schematic model of the arc is given in figure 1 . The general expression for the centre-line intensity $I(0)\left(\mathrm{s}^{-1} \mathrm{sr}^{-1}\right)$ and the normalised velocity distribution $P(v)$ of the molecular beam flux is given by

$$
I(0) P(v) \mathrm{d} v \mathrm{~d}^{2} \omega=\left(\int_{0}^{L_{\mathrm{p} 1}} \dot{n}(z, v) T(z, v) A \mathrm{~d} z\right) \mathrm{d} v \mathrm{~d}^{2} \omega / 4 \pi
$$

with $\mathrm{A}$ the area of the sampling orifice, $L_{\mathrm{p} 1}$ the arc length, $\dot{n}(z, v)$ $\left(\mathrm{m}^{-3} \mathrm{~s}^{-1}\left(\mathrm{~ms}^{-1}\right)^{-1}\right)$ the production rate of the molecular beam species per velocity interval and per unit of volume and $T(z, v)$ the transmission probability of a particle with velocity $v$ through the plasma slice between the production region $z$ and the end anode $(z=0)$. In general these quantities are determined by collisions between neutrals, ions and electrons.

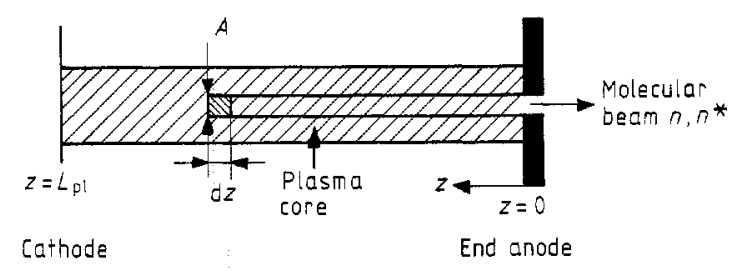

Figure 1. Model of the hollow cathode are and the sampling orifice.

In many cases equation (1) can be simplified considerably in order to get more insight in the process of molecular beam sampling. A useful variable is the view depth in the plasma $\lambda(v)$. It determines the region in the plasma that contributes effectively to the molecular beam formation. When $T\left(L_{\mathrm{pl}}, v\right) \ll 1$, which holds for all of our experimental conditions, the view depth $\hat{\lambda}(v)$ is given by

$$
\lambda(v)=\int_{0}^{\infty} T(z, v) \mathrm{d} z
$$

To a first approximation we assume a homogeneous plasma, i.e. $\dot{n}(z, v)=\dot{n}(v)$, in the region contributing to the production of the molecular beam. The mean velocity $\langle v\rangle$ of the molecular beam particles produced in the plasma is given by

$$
\langle v\rangle=\int_{0}^{\infty} v \dot{n}(v) \mathrm{d} v / \int_{0}^{\infty} \dot{n}(v) \mathrm{d} v .
$$


If the view depth is a smooth and slowly varying function in the velocity range around $\langle v\rangle$, we can approximate equation (1) by

$$
I(0) \mathrm{d}^{2} \omega \approx \frac{\dot{n}(\langle v\rangle) \lambda(\langle v\rangle) A \mathrm{~d}^{2} \omega}{4 \pi}
$$

If we apply these approximations to the case of an effusive source with only one species, i.e. ground state atoms, we find

$$
\begin{gathered}
\dot{n}(\langle v\rangle) \approx \frac{1}{2} n_{n}^{2}\langle g\rangle Q \\
T(z,\langle v\rangle) \approx \exp -\left(n_{n} Q z\langle g\rangle /\langle v\rangle\right) \\
I(0) \mathrm{d}^{2} \omega \approx \frac{n_{\mathrm{n}}\langle v\rangle A}{8 \pi} \mathrm{d}^{2} \omega
\end{gathered}
$$

with $Q$ the velocity independent production cross section, $\langle g\rangle$ the mean relative velocity and $n_{n}$ the density. Within a factor of two equation (5) is the correct result.

We now discuss the production processes for fast ground state and metastable atoms.

\subsection{Ground state atoms}

Fast ground state atoms are produced by collisions between ions and thermal neutrals (ground state atoms). Two processes have to be taken into account, i.e. elastic collisions and charge exchange collisions. The latter process is most important. The transmission term $T(z, v)$ is determined by collisions with ions, thermal neutrals and electrons (ionisation). A more detailed theoretical description is given by Theuws et al (1977a). We now only review the results. The subscripts $n, e$ and $i$ refer to thermal neutrals, electrons and ions, respectively. Parameters without a subscript refer to fast ground state atoms.

To a first order approximation, the centre-line intensity of fast ground state atoms is then given by

$$
\begin{gathered}
I(0) \mathrm{d}^{2} \omega=n_{\mathrm{n}} n_{\mathrm{i}} A\left\langle v_{\mathrm{i}}\right\rangle\left(Q_{\mathrm{el}}+Q_{\text {exch }}\right) \lambda(\langle v\rangle) \mathrm{d}^{2} \omega / 4 \pi \\
\text { with } \lambda(\langle v\rangle)=\left(n_{\mathrm{i}} Q_{\mathrm{i}} 2^{1 / 2}+n_{\mathrm{e}}\langle Q v\rangle_{\text {ion }}\langle v\rangle^{-1}+n_{\mathrm{n}} Q_{\mathrm{n}}\right)^{-1},
\end{gathered}
$$

where $Q_{\text {el }}$ and $Q_{\text {exoh }}$ are the total cross sections for elastic (hard sphere) collisions and charge exchange collisions, respectively, $Q_{\mathrm{i}}=Q_{\mathrm{el}}+Q_{\text {exch }}$ is the total cross section for ion-atom collisions, $\langle Q v\rangle_{\text {ion }}$ is the cross section-velocity product for ionisation weighted over the velocity distribution of the electrons, $n_{\mathrm{n}}, n_{\mathrm{i}}$ and $n_{e}$ are the number densities of the different species, $Q_{n}$ is the cross section between fast ground state atoms and thermal neutrals, and $\left\langle v_{i}\right\rangle$ is the mean velocity of the ions.

A suitable parameter is the degree of ionisation $\beta$ of the plasma, given by

$$
\beta=n_{\mathrm{i}} /\left(n_{\mathrm{i}}+n_{\mathrm{n}}\right)
$$

which varies between 0 and 1 . In figure 2 we have plotted a normalised intensity $I(0) / I(0)_{\text {ref }}$ as a function of $\beta$, neglecting the ionisation term in $\lambda(\langle v\rangle)$. The reference intensity $I(0)_{\text {ref }}=$ $\left(n_{\mathrm{i}}+n_{\mathrm{n}}\right)\left\langle v_{\mathrm{i}}\right\rangle A / 4 \pi$ corresponds to the intensity of an effusive source with heavy particle density $\left(n_{1}+n_{n}\right)$ and temperature $T_{i}$. At $\beta=0.35$ we see a maximum in the reduced intensity. However, in a wide interval $0.1 \leqslant \beta \leqslant 0.8$ this reduced intensity only varies by a factor of two.

In equation (6) we can distinguish two limiting cases. At low $\beta$-values, i.e. a low electron temperature $T_{e}$, the ions are the limiting factor in the production function and $I(0) / I(0)_{\text {ref }}$ is given by

$$
I(0) / I(0)_{\text {ref }}=\beta\left[\left(Q_{\mathrm{el}}+Q_{\text {exch }}\right) / Q_{\mathrm{n}}\right] .
$$

For high $\beta$-values (high $T_{e}$ ) the neutral density is the limiting factor and we find

$$
I(0) / I(0)_{\mathrm{ref}}=(1-\beta)\left(2^{-1 / 2}\right)
$$

Typical values for argon are $Q_{\mathrm{el}}=0.17 \mathrm{~nm}^{2}, Q_{\text {exch }}=0.47 \mathrm{~nm}^{2}$, and $Q_{\mathrm{n}}=0.2 \mathrm{~nm}^{2}$ at $1 \mathrm{eV}$ (Theuws et al 1977a).

With increasing values of $\beta$ the electron temperature will increase and the influence of collisions with electrons on the transmission loss (equation (6)) will have to be taken into account, resulting in a lower value of the reduced centre-line intensity $I(0) / I(0)_{\text {ref }}$ than given in figure 2 .

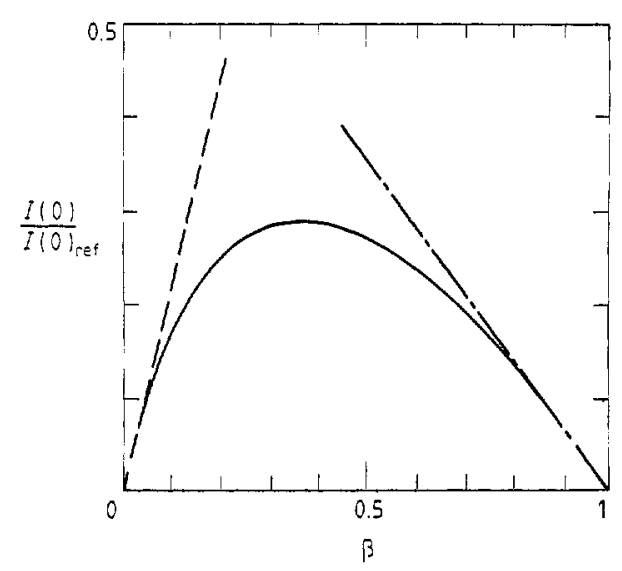

Figure 2. Model calculations of the normalised centre-line intensity $I(0) / I(0)_{\text {ref }}$ of fast ground state atoms, with $I(0)_{\text {ref }}=\left(n_{\mathrm{i}}+n_{\mathrm{n}}\right)\langle v\rangle A / 4 \pi$. The broken line (equation (8)) and the chain line (equation (9)) represent the limiting cases of a low and a high degree of ionisation $\beta$, respectively, with $\beta=n_{\mathrm{i}} /\left(n_{\mathrm{n}}+n_{\mathrm{i}}\right)$.

\subsection{Metastable atoms}

For the production of fast metastable atoms two processes are considered. Firstly a collision of a ground state atom with an ion (charge exchange or elastic collision) and successive excitation by a collision with an electron. Secondly, we consider a collision of a metastable atom with an ion with exchange of charge and excitation. Model calculations show that the first process is the dominating process of molecular beam formation (Theuws et al 1979a, Theuws 1981) and we shall only discuss this process.

The first process can be related directly to our calculations on the sampling of fast ground state atoms. For fast ground state atoms the view depth $\lambda(v)$ into the plasma is determined mainly by collisions with ions and neutrals and for $T_{\mathrm{e}}<3 \mathrm{eV}$ ionisation can be neglected.

For metastable atoms however, the view depth $\lambda_{n^{*}}\left(v_{n^{*}}\right)$ is fully determined by excitation to higher states and ionisation by collisions with electrons, and is given by

$$
\lambda_{n^{*}}\left(v_{n^{*}}\right)=v_{n^{*}} /\left(n_{\mathrm{e}}\langle Q v\rangle_{\text {ion, } n^{*}}\right),
$$

with $n_{\mathrm{e}}\langle Q v\rangle_{\text {ion, } n^{*}}$ the rate constant for the loss of metastable atoms and $v_{n^{*}}$ the velocity of the metastable atom. For the noble gases the relation $\lambda_{n^{*}} \ll \lambda$ holds for all plasma conditions considered in our experiments. In this first model the centre-line intensity $I(0)_{n^{*}}\left(\mathrm{~s}^{-1} \mathrm{sr}^{-1}\right)$ is directly proportional to the centreline intensity $I(0)$ of the fast ground state atoms as given by

$$
I(0)_{n^{*}} \mathrm{~d}^{2} \omega=I(0) P_{\text {exc }}\left(z \leqslant \lambda_{n^{*}}\right) \mathrm{d}^{2} \omega,
$$

with $P_{\text {exc }}\left(z \leqslant \lambda_{n^{*}}\right)$ the probability that a fast ground state atom will be excited to a metastable state in the plasma slice with thickness $\lambda_{n^{*}}$ in front of the sampling orifice in the end anode. Hereby we implicitly assume that $T_{n^{*}}\left(z, v_{n^{*}}\right)=1$ for $z \leqslant \lambda_{n^{*}}$ and zero elsewhere. The probability $P_{\text {exc }}\left(z \leqslant \lambda_{n^{*}}\right)$ is equal to

$$
P_{\text {exc }}\left(z \leqslant \lambda_{n^{*}}\right)=\tau_{n^{*}} n_{\mathrm{e}}\langle Q v\rangle_{\mathrm{exc}}
$$


with $\tau_{n^{*}}=\lambda_{n^{*}} / v_{n^{*}}$ the transit time of the fast metastable atom through the plasma slice with thickness $\lambda_{n^{*}}$ and $n_{e}\langle Q v\rangle_{\text {exc }}$ the excitation rate of the fast ground state atoms. We can now write equation (11) as

$$
I(0)_{n^{*}} \mathrm{~d}^{2} \omega=I(0)\left[\langle Q v\rangle_{\text {exc }} /\langle Q v\rangle_{\text {ion }, n^{*}}\right] \mathrm{d}^{2} \omega,
$$

where we have used $v_{n^{*}}=v$, i.e. we neglect the momentum of the electron in the momentum balance.

For the noble gases with their metastable levels far above the ground state $\left(E^{*} \approx 11.6 \mathrm{eV}\right.$ for argon) and the close lying group of ten $2 \mathrm{p}$ levels (Paschen notation) at an energy difference $\Delta E$ $(\Delta E \approx 1.6 \mathrm{eV}$ for argon) the rate constants for excitation and ionisation of metastable atoms will strongly depend on the electron temperature in the range $T_{t}=1-5 \mathrm{eV}$. We can thus expect that conditions of high $T_{\mathrm{e}}$ are favourable for high intensities of metastable atoms. For argon we expect $I(0)_{n^{*}} / I(0)=2 \times 10^{-5}, \quad 3 \times 10^{-4}, \quad 9 \times 10^{-4}$ for electron temperatures $T_{\mathrm{e}}=2,3$ and $4 \mathrm{eV}$, respectively (Theuws 1981, Ferreira and Delcroix 1975, 1978).

\section{Experimental set-up}

\subsection{Hollow cathode arc}

The essential part of a hollow cathode arc is the hollow cathode. This is a tube with a large length to diameter ratio $(\approx 40)$ and is fabricated of a refractory metal ( $\mathrm{Ta}$ or $\mathrm{W}$ ), that can withstand high temperatures $(2500-3000 \mathrm{~K})$. Gas feed takes place through the cathode. Detailed theoretical studies on the operation of the hollow cathode have been performed by Ferreira and Delcroix (1978). Electrons are emitted by the hot cathode through thermoemission and are accelerated in a positive sheath at the inner cathode wall. The incoming neutral gas is excited and ionised by these high energy $(10-20 \mathrm{eV})$ electrons. A large amount of this energy is returned to the cathode by ions mainly and probably metastables and photons. In this way the cathode is heated to the temperature needed for sufficient thermoemission. The most striking visible feature for these effects is the bright spot several diameters upstream from the cathode exit, the so-called hot spot.

Figure 3 shows a detailed design of the source head, i.e. cathode and ring anode. The cathode support (copper), the ring anode (strainless steel tube with 2.3 and $3 \mathrm{~mm} \mathrm{I/D} \mathrm{and} \mathrm{O} / \mathrm{D}$, respectively, with a copper ring added) and the end anode (stainless steel or tantalum on a copper base) are all water cooled. Special attention has been paid to appropriate screening to avoid spurious current paths. Electrical breakdown in the gas feed line (pressure $1.3-6.7 \mathrm{kPa}(10-50$ Torr)) is avoided by a long insulation gap. Starting of the arc is performed by a small spark discharge $(4 \mathrm{kV}, 20 \mathrm{~mA})$ between the cathode and the ignition electrode $(0.5 \mathrm{~mm}$ diameter tungsten wire). During the start up $300 \mathrm{~V}$ is applied between ring anode and cathode to supply sufficient heating of the cathode by ion bombardment until steady state operation is reached. A series resistor is used to compensate for the negative voltage-current characteristic of the arc. Operating the arc on $\mathrm{Ne}$ or $\mathrm{Kr}$ is achieved by starting the arc on Ar. The Ar flow is then lowered and compensated by an additional $\mathrm{Ne}$ or $\mathrm{Kr}$ flow until there is a pure $\mathrm{Ne}$ or $\mathrm{Kr}$ flow.

The external plasma is confined by a weak axial magnetic field $\left(B_{0}<0.05 \mathrm{~T}\right)$. It is characterised by a high ionisation degree and densities of the order of $10^{18}-10^{22} \mathrm{~m}^{-3}$, depending on the flow rate through the cathode and the distance from the cathode exit. The electron temperature varies between 2 and $6 \mathrm{eV}$ with the ion temperature $T_{\mathrm{i}} \leqslant T_{\mathrm{e}}$.

Typical operating currents range from $I_{\mathrm{a}}=5-20 \mathrm{~A}$. The operating voltage depends both on the gas and the cathode material. Typical values are $60-90 \mathrm{~V}$ for $\mathrm{Ne}(\mathrm{W}), 20-50 \mathrm{~V}$ for $\mathrm{Kr}(\mathrm{Ta})$, and 20-70 V for $\mathrm{Ar}(\mathrm{Ta})$.
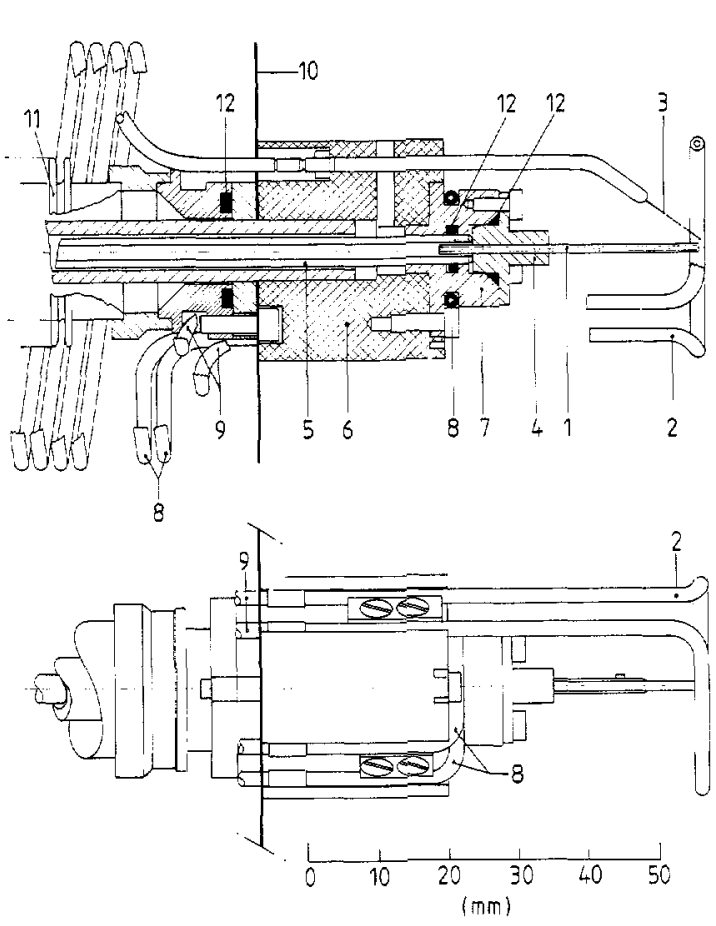

Figure 3. Cross section and side view (rotation $90^{\circ}$ ) of construction of the source head. 1 , hollow cathode, tungsten, 1 and $2 \mathrm{~mm}$ inner and outer diameter, 20-30 $\mathrm{mm}$ external length; 2 , ring anode, stainless steel, 2.4 and $3.0 \mathrm{~mm}$ inner and outer diameter; 3, ignition electrode, tungsten, $0.5 \mathrm{~mm}$ diameter; 4 , hollow cathode mount, copper; 5 , gas feed line, glass; 6 , insulator with degassing channel, machinable glass ceramic; 7 , hollow cathode support (water-cooled), copper; 8 , water cooling and electrical connection of cathode support, stainless steel, 2.4 and $3.0 \mathrm{~mm}$ inner and outer diameter; 9, water cooling and electrical connection of ring anode, stainless steel, 2.4 and $3.0 \mathrm{~mm}$ inner and outer diameter; 10, insulator, pertinax; 11 , bellows for aligning hollow cathode to sampling orifice, stainless steel; 12, $\mathbf{O}$-ring seals. The water cooling coils are insulated with Teflon tubing. All parts not mentioned are stainless steel.

In all our experiments the inner and outer diameters of the cathode are $1 \mathrm{~mm}$ and $2 \mathrm{~mm}$, respectively. The external length of the hollow cathode is $20-30 \mathrm{~mm}$. The normal procedure is to start with a tube of $100 \mathrm{~mm}$, which is cut off when a hole is burnt in the cathode at the hot spot. The loss of cathode material is less using this method. The diameter of the plasma column is typically $2 \mathrm{~mm}$ resulting in current densities of $10^{6} \mathrm{~A} \mathrm{~m}^{-2}$.

\subsection{Temperatures and life time of the cathode}

To perform scattering experiments we need a molecular beam source which can operate for at least 24 hours without major changes in its characteristics. The main limitation of operating a HCA stems from the life time of the cathode, which has a temperature close to the melting point and evaporates a lot of material. We have measured the temperature profile of the cathode, using a pyrometer that is calibrated with a tungsten ribbon lamp. Absolute accuracy of the measurements is typically $10 \%$, relative accuracy is of the order of $0.7 \%$. For tungsten we have performed absolute measurements. For comparison we have also measured temperature profiles for tantalum. These measurements show perfect agreement with the results of Delcroix and Trindade (1974), both in absolute value and in general behaviour. For $\mathrm{Ta}$ the temperature at the hot spot 
varies from $2400-2700 \mathrm{~K}$ and depends on the arc current, the magnetic induction and the flow rate through the cathode. Figure 4 shows temperature profiles for different gas and cathode material combinations. The measured temperatures for $\mathrm{W}$ are higher as compared to $\mathrm{Ta}$ in agreement with the Richardson equation. The highest temperatures are measured for Ne. The temperature increases with increasing arc current and the distance between the hot spot and the cathode exit decreases with increasing flow rate. These results are similar to the results of Delcroix and Trindade (1974).

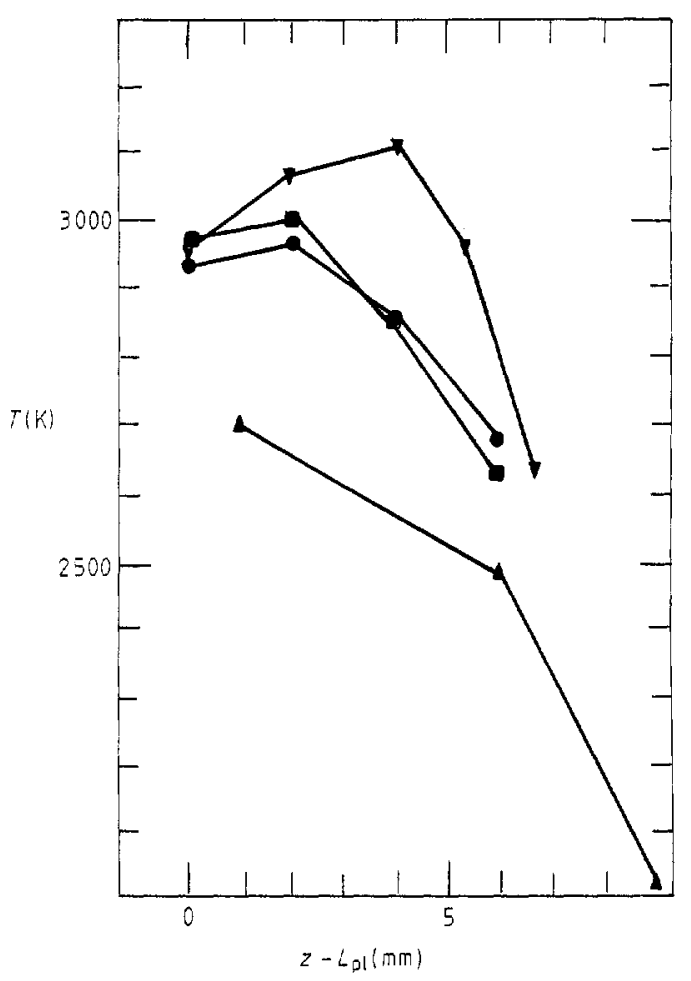

Figure 4. The temperature profile of the hollow cathode for different gas and cathode material combinations. The arc current is $I_{\mathrm{a}}=10 \mathrm{~A}$ and the peak magnetic induction is $B_{0}=1.8 \times 10^{-2} \mathrm{~T}$. For Ar-Ta, Ar-W and $\mathrm{Kr}-\mathrm{W}$ the flow rate is $\dot{N}=5.5 \times 10^{18} \mathrm{~s}^{-1}$, for Ne-W the flow rate is $\dot{N}=8.4 \times$ $10^{18} \mathrm{~s}^{-1} .-\nabla-, \mathrm{Ne}-\mathrm{W} ;-\mathbf{-}-\mathrm{Ar}-\mathrm{W} ;-\mathbf{-}, \mathrm{Kr}-\mathrm{W} ;-\mathbf{-}-$, Ar-Ta.

Table 1 lists typical results for life times for the gases $\mathrm{Ne}, \mathrm{Ar}$ and $\mathrm{Kr}$ and the cathode materials $\mathrm{Ta}$ and $\mathrm{W}$. The combination $\mathrm{Ne}(W)$ shows the shortest life time as can be expected from the measured temperatures. However, a time of $10-15 \mathrm{~h}$ is still sufficient for many experiments.

In our experiments we observe that the life time of the cathode decreases drastically when impurities can enter the cathode. Small air leaks in the order of $10^{-4}$ of the flow rate of

Table I. Life time (h) of cathode tubes ( $1 \mathrm{~mm}$ and $2 \mathrm{~mm}$ inner and outer diameter, respectively) for different combinations of gas and cathode material.

\begin{tabular}{llll}
\hline & $\mathrm{Ne}$ & $\mathrm{Ar}$ & $\mathrm{Kr}$ \\
\hline $\mathrm{Ta}$ & - & 40 & 40 \\
$\mathrm{~W}$ & 15 & 40 & 40 \\
\hline
\end{tabular}

the noble gas already decreases the life time of the cathode by a factor of ten. In these cases we also find the growth of a constriction near the hot spot which finally blocks off the cathode. Without impurities we also find such constrictions but to a far lesser degree. Also materials like Teflon in the vacuum chamber, which start to outgas when heated by the radiation of the hot cathode tube, result in a sharp decrease of the life time of the cathode and cause the growth of similar constrictions inside the cathode tube.

\subsection{The time-of-flight method}

All measurements have been performed in a time-of-fight (TOF) machine. A detailed description is given elsewhere (Beijerinck et al 1974).

For ground state particles we use a cross beam ioniser, a quadrupole mass filter and an electron multiplier (beam density detector). Single ion counting is used. The overall detection efficiency has been calibrated by using an effusive source (Beijerinck et al 1974).

The metastable atoms are detected by Auger ejection of an electron from a stainless steel surface, followed by an electron multiplier, resulting in a detection efficiency that is independent of the initial velocity of the metastable atom (beam flux detector)

For a low intensity, thermal energy beam of metastable argon we have determined a secondary electron yield $\gamma_{\mathrm{A} \tau^{*}}=0.1$ (Theuws 1981, Theuws et al 1982). The scaling of the $\gamma_{n^{*}}$ values for the other noble gases is in reasonable agreement with Borst (1971). However, it is possible that the metal detector surface behaves differently with respect to $\gamma_{n}$, when it is bombarded by a high intensity flux of ground state and metastable atoms in the $\mathrm{eV}$ translational energy range and photons from the hollow cathode arc (during the open time of the chopper). A careful analysis of the plasma parameters of the long arc configuration (see $\S 4.2$ ), as determined from our beam measurements, strongly suggests that $\gamma_{\mathrm{Ar}^{*}} \approx 1$ for the plasma source measurements (Theuws 1981). Throughout this paper we have assumed a secondary electron yield equal to unity for $\mathrm{Ne}, \mathrm{Ar}$ and $\mathrm{Kr}$, and the data given are thus lower bounds for the actual intensity.

\section{Experimental results}

\subsection{Data analysis}

A typical TOF spectrum of ground state atoms is given in figure 5. The ratio of the full width at half-maximum and the position of the maximum is typically equal to 0.7 , which can be compared to the value 0.83 for a Maxwell-Boltzmann distribution and to 0.56 for a supersonic distribution with a speed ratio $S=3$. Because a least squares analysis of the measured spectra with obvious model functions like e.g. a supersonic distribution (Anderson 1974) or a Maxwell-Boltzmann distribution does not give satisfactory results, we have followed a different procedure to determine $I(0)$ and $T$.

The intensity has been calculated by summation of the total number of counts in the TOF spectrum. For a beam flux detector (metastable atoms) this result is directly proportional to $I(0)_{n^{*}}$. With a beam density detector we first have to correct the contents of each channel for the velocity dependency of the detector efficiency.

The temperature has been derived from the mean energy $\frac{1}{2} m\left\langle v^{2}\right\rangle$ of the beam density, using $\frac{3}{2} k T=\frac{1}{2} m\left\langle v^{2}\right\rangle$ which gives the correct relation with the plasma temperature in the case of a Maxwell-Boltzmann velocity distribution in the beam.

4.2. Definition of a short arc

As we have seen in $\$ 2$ the intensity is proportional to the 


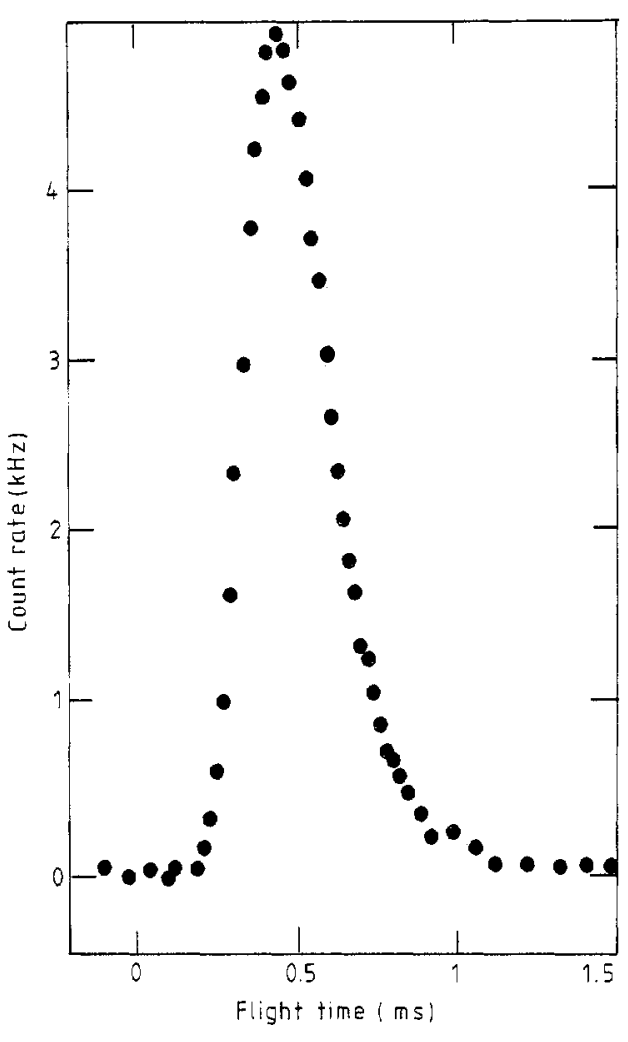

Figure 5. A time-of-fight spectrum of fast ground state atoms for argon in the short arc configuration. The flight path is $1.65 \mathrm{~m}$.

density and for optimum results as a molecular beam source we are interested in a high density plasma. The density in the vacuum chamber of the plasma source is determined by the flow rate and the pumping speed. With a pumping speed of $701 \mathrm{~s}^{-1}$ and flow rates ranging from $\dot{N}=0.3 \times 10^{19}-3 \times$ $10^{19} \mathrm{~s}^{-1}$ the densities are of the order of $10^{20} \mathrm{~m}^{-3}$. The neutral and ion densities in the plasma far downstream of the cathode are fully determined by this density in the vacuum chamber. This region is referred to as the so-called long arc configuration. Results on the long arc configuration and on the use of this experimental method as a plasma diagnostic are described elsewhere (Theuws 1981).

Gas feed takes place through the cathode and we can expect much higher densities close to the cathode exit. The number density at the exit is determined by the flow rate $\hat{N}$ and the flow velocity at the exit, which is equal to the local velocity of sound. To a first approximation we can write

$$
\dot{N}=0.5 \ln \alpha\left(T_{\text {cath }}\right)\left(\frac{\pi}{4} d_{\text {cath }}^{2}\right),
$$

with $\alpha(T)=(2 k T / m)^{1 / 2}, T_{\text {cath }}$ the cathode temperature and $d_{\text {cath }}$ the diameter of the cathode exit. Equation (14) holds exactly for the flow of a monatomic gas through an ideal sonic nozzle (Anderson 1974). For argon and the experimental values $\dot{N}=$ $10^{19} \mathrm{~s}^{-1}, T_{\text {cath }}=3000 \mathrm{~K}, d_{\text {cath }}=1 \mathrm{~mm}$ we find $n=2 \times 10^{22} \mathrm{~m}^{-3}$, which is two orders of magnitude higher than the number density in the vacuum chamber. The neutral and ion densities in the plasma close to the cathode exit will scale with this calculated number density. The configuration with the sampling orifice (and thus the end anode) in this region of the plasma column is called the short arc configuration and is most efficient as a molecular beam source (Theuws et al 1977b, 1979b).

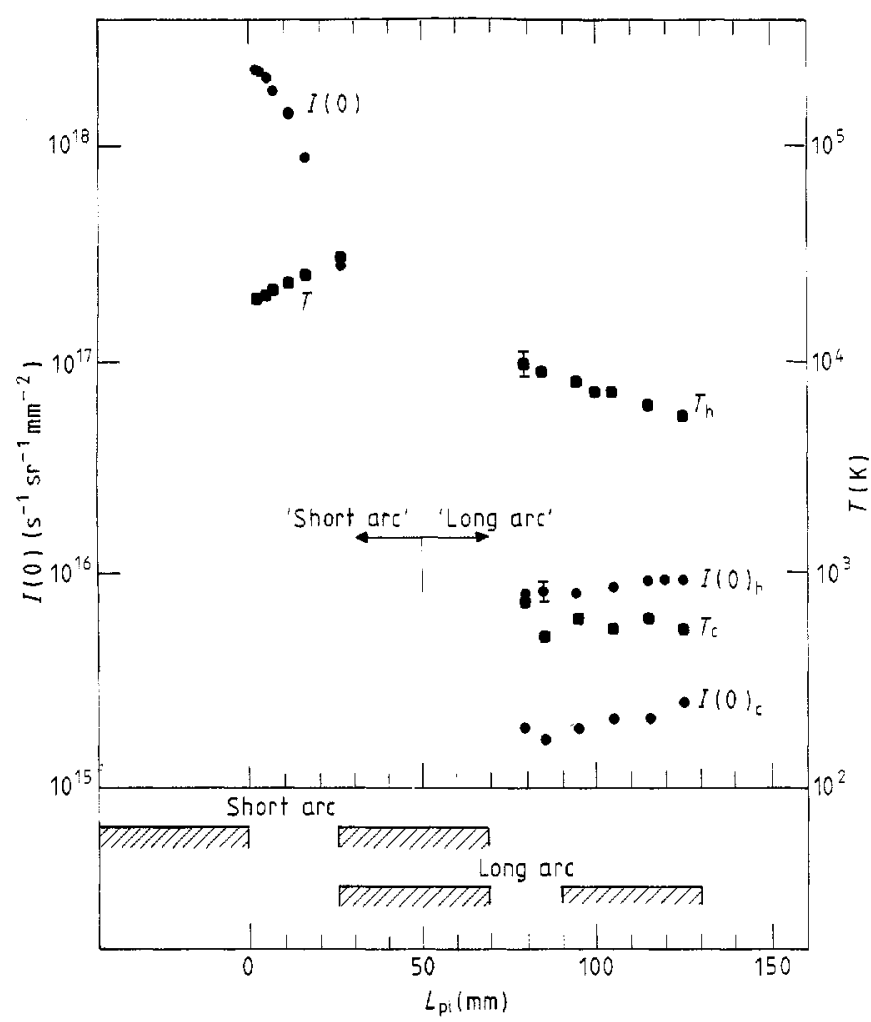

Figure 6. The measured centre-line intensity and temperature of fast ground state atoms of argon as a function of the arc length $L_{\mathrm{pl}}$, at an arc current $I_{\mathrm{a}}=10 \mathrm{~A}$ and a peak magnetic induction $B_{0}=3.6 \times 10^{-2} \mathrm{~T}$. The lower part shows the configuration of magnetic coils in the long arc (bottom) and in the short arc configuration (top), respectively. In the long arc configuration a bimodal velocity distribution function is used to describe the TOF spectra. The two contributions are indicated with the subscripts $h(o t)$ and $c(o l d)$.

Figure 6 shows the intensity $I(0)$ as a function of the length $L_{\mathrm{pl}}$ of the arc. We observe that with increasing distance $z^{\prime}=$ $\left(L_{\mathrm{pl}}-z\right)$ from the cathode exit the number density (and thus the intensity) decreases much slower than predicted by $n\left(z^{\prime}\right) / n(0) \approx\left[z^{\prime} /\left(0.4 d_{\text {cath }}\right)\right]^{-2}$, which holds for a free supersonic expansion of a monoatomic gas for $z^{\prime} / d_{\text {cath }} \geqslant 3$ (Beijerinck and Verster 1981). By ion-atom collisions the ground state neutrals are confined on the axis during several characteristic times for diffusion over a distance equal to the plasma radius. If we take into account the high flow velocity (velocity of sound at the cathode exit)) we can convert this time into a distance. The equilibrium of the neutral density inside the arc with the neutral density in the vacuum chamber is thus only reached far downstream of the cathode exit.

From figure 6 we derive a characteristic length of the short arc region of $70 \mathrm{~mm}$, which should be compared to $5 \mathrm{~mm}$ as predicted by a free expansion from a sonic nozzle. Calculations with the above mentioned qualitative model result in $60 \mathrm{~mm}$, and are in good agreement with our experimental results (Theuws 1981). Application of these same criteria to a hollow cathode arc with fully different dimensions also give a good prediction of this transition of the short arc region to the long arc region (Timmermans et al 1981).

\subsection{Ground state atoms}

Figure 7 shows the intensity $I(0)$ of fast ground state atoms for Ar as a function of the flow rate $\dot{N}$ through the cathode for an 


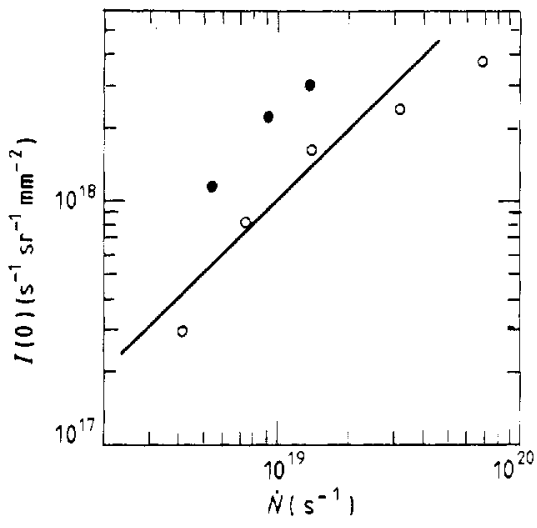

Figure 7. Measured centre-line intensity of fast ground state atoms for argon for the arc described in $\S 3$ (full circles) and a different model with a large pumping speed of $10001 \mathrm{~s}^{-1}$ (open circles). The straight line is a model calculation with equation (6) (see $\S 4.3$ ). Arc current $I_{\mathrm{a}}=10 \mathrm{~A}$ and $B_{0}=2.1 \times 10^{-2} \mathrm{~T}$. The arc length is $L_{\mathrm{pl}}=8 \mathrm{~mm}$.

arc current $I_{\mathrm{a}}=10 \mathrm{~A}$ and a magnetic induction $B_{0}=2.1 \times$ $10^{-2} \mathrm{~T}$. The length of the plasma column is $L_{\mathrm{pl}}=8 \mathrm{~mm}$. In figure 7 we also give results for a plasma source operated in a vacuum chamber with a pumping speed of approximately $1000 \mathrm{~s}^{-1}$. Within a factor of two we see no difference in intensity with the source described in this paper with a $701 \mathrm{~s}^{-1}$ pump. For both sources we conclude that the intensity is proportional to the flow rate $\dot{N}$.

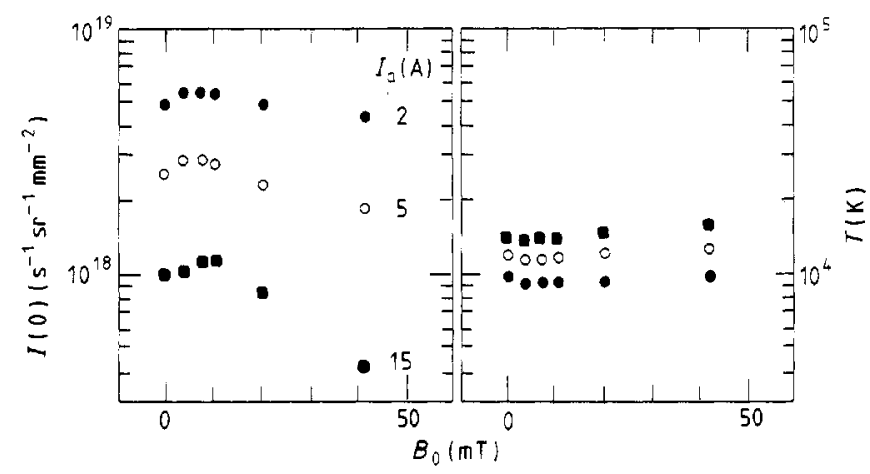

Figure 8. Measured centre-line intensities and temperatures of fast ground state argon atoms as a function of the peak magnetic induction $B_{0}$ and the arc current $I_{\mathrm{a}}$. The arc length is $L_{\mathrm{pl}}=8 \mathrm{~mm}$.

Figure 8 shows the intensity of fast ground state atoms for $\mathrm{Ar}$ as a function of $B_{0}$ for different arc currents $I_{\mathrm{a}}$ and a flow rate $\dot{N}=3.2 \times 10^{19} \mathrm{~s}^{-1}$, again for $L_{\mathrm{pl}}=8 \mathrm{~mm}$. The most distinguishing feature is the sharply decreasing intensity with increasing arc current. Extensive measurements (Theuws et al 1977a, Theuws 1981) show that this is in qualitative agreement with our model of equation (9) for a high degree of ionisation.

The temperature for argon increases slightly with increasing arc current as can be expected from the extra energy input from the electrons.

For $\mathrm{Kr}$ the intensity is approximately a factor two lower than for $\mathrm{Ar}$ for comparable plasma conditions while the temperature is approximately equal.
Using the maximum normalised intensity $I(0) / I(0)_{\mathrm{ref}}=0.29$ of equations (6), (8) and (9) we have calculated the maximum centre line intensity $I(0)$ as a function of the flow rate $\dot{N}$, using equation (14) to estimate the number density $\left(n_{\mathrm{i}}+n_{\mathrm{n}}\right)$. The result is given in figure 7 . We see a fair agreement with the experimental results.

\subsection{Metastable atoms}

Figure 9 shows the intensity and temperature of metastable atoms for Ar as a function of the flow rate $\dot{N}$ for different arc currents $I_{\mathrm{a}}$ and $B_{0}=3.6 \times 10^{-2} \mathrm{~T}$. Both the intensity and temperature are approximately constant over the whole range of flow rates while the intensity decreases and the temperature increases with increasing arc current. Comparing this with the behaviour of the fast ground state atoms we can conclude that the decrease of the intensity as a function of the arc current is less for metastable atoms. This results from an increasing electron temperature $T_{\mathrm{e}}$ with increasing arc current (equation (13)).

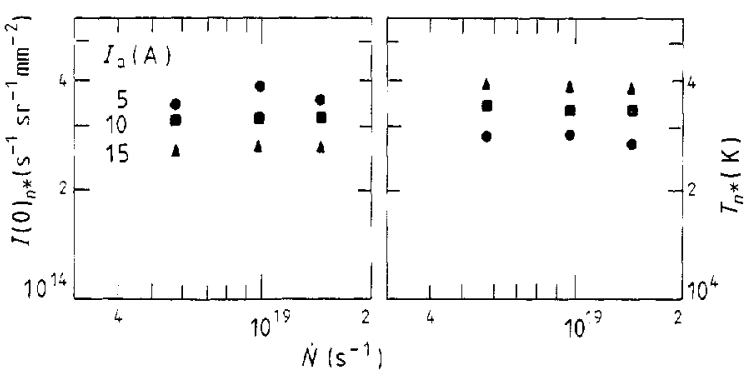

Figure 9. Measured centre-line intensities and temperatures of metastable argon atoms as a function of the flow rate $\dot{N}$ through the cathode, for three values of the arc current $I_{\mathrm{a}}$.

We have also performed measurements on $\mathrm{Ne}^{*}$ and $\mathrm{Kr}^{*}$. The temperatures are approximately equal for all three gases. For $\mathrm{Ne}^{*}$ the intensities are approximately a factor two higher than for $\mathrm{Ar}^{*}$. For $\mathrm{Kr}^{*}$ the intensity is a factor ten lower than for Ar*. For all gases a secondary electron yield $\gamma_{n^{*}}=1$ has been assumed, and the intensities given are thus lower limits (see § 3.3).

From the ratio of the intensity of the metastable atoms and the intensity of the ground state atoms we can deduce an electron temperature $T_{\mathrm{s}} \approx 3 \mathrm{eV}$ for argon (Theuws 1981). This is in fair agreement with $T_{\mathrm{e}}$ measurements with Thomson scattering in a comparable argon plasma in a different HCA (Pots 1979).

For $\mathrm{Ne}^{*}$ we have investigated the beam composition by optical pumping with a dye-laser in combination with a time-offlight analysis of the optically pumped beam (Kroon et al 1981a, b). The experimental result is a population ratio $\mathrm{Ne}^{*}\left({ }^{3} \mathrm{P}_{2}\right) / \mathrm{Ne}^{*}\left({ }^{3} \mathrm{P}_{0}\right)=(6.7 \pm 1)$, which is in agreement with the ratio of statistical weights (5) and the Boltzmann factor of typical group configuration temperatures as determined from line intensity measurements (Van der Sijde 1972).

\section{Conclusions}

We have developed a reliable molecular beam source for both fast ground state atoms and fast metastable atoms with a high centre-line intensity and a broad velocity distribution. The experimental results on the centre-line intensity are in fair agreement with model calculations of the process of molecular beam sampling of the plasma. The beam source is small in size 
and easy to operate. The long term stability of $10-40 \mathrm{~h}$ is sufficient for most scattering experiments (Van der Kam 1981).

For elastic scattering experiments, e.g. the measurement of the total cross section, the mixed beam of fast atoms in the ground state and in metastable states does not limit the applicability of the source. The detector used for the metastable atoms, i.e. Auger ejection of an electron from a metal surface, is insensitive for ground state atoms in the translational energy range considered. The ionisation detector for ground state atoms also ionises the metastable atoms. However, due to the small ratio $I(0)_{n^{*}} / I(0)$ these effects are negligible.

For inelastic or reactive scattering experiments we have to consider the effect of a mixed beam more carefully. For the noble gas atoms, however, with their metastable levels far above the ground state, most reactive channels will be opened by their internal electronic energy, and in many cases the contribution of fast ground state atoms to reactive scattering can be neglected. For experiments with a single metastable state optical pumping with a dye laser can be applied to eliminate the other metastable state (Kroon et al 1981a, b).

\section{References}

Anderson J B 1974 Molecular beams from nozzle sources Molecular Beams and Low Density Gasdynamics ed. P P Wegener (New York: Dekker) p 1

Anderson J B, Andres R P and Fenn J B 1965 High intensity and high energy molecular beams, Advances in Atomic and Molecular Physics ed. D R Bates and I'Estermann vol. 1 (New York: Academic) p 345

Beijerinck H C W, Moonen R G J M and Verster N F 1974 Calibration of a time-of-flight machine for molecular beam studies

J. Phys. E: Sci. Instrum. 731

Beijerinck H C W and Verster N F 1981 Absolute intensities and perpendicular temperatures of supersonic beams of polyatomic gases

Physica C 111327

Bickes R W Jr, Newton K R, Hermann J M and Bernstein R B 1976 Utilization of an arc-heated jet for production of supersonic seeded beams of atomic nitrogen

J. Chem. Phys. 643648

Borst W L 1971 Secondary electron yields from a Cu-Be-O surface by thermal $\mathrm{CO}, \mathrm{N}_{2}$ and noble gas metastables

Rev. Sci. Instrum. 421543

Delcroix $\mathrm{J} \mathrm{L}$ and Trindade A R 1974 Hollow cathode arcs $A d v$. in Electr. and Electron. Phys. vol. 35 (New York: Academic) p 87

Ferreira C M and Delcroix J L 1975 Théorie de la decharge d'arc à cathode creuse II. Bilan de métastables à l'intérieur de la cathode. Application à l'argon

J. Physique 361241

Ferreira C M and Delcroix J L 1978 Theory of the hollow cathode arc

J. Appl. Phys. 492380

Gillen K T, Saxon R P, Lorents D C, Ice G E and Olson R E 1976 Elastic scattering measurements of the $\operatorname{Ar}_{2}^{*}\left({ }^{3} \Sigma_{u}^{+}\right)$well depth

J. Chem. Phys. 641925

Kroon J P C, Beijerinck H C W and Verster N F 1981a TOF analysis of an optically pumped metastable neon beam J. Chem. Phys. 746528

Kroon J P C, Beijerinck $\mathrm{H} \mathrm{C} \mathrm{W,} \mathrm{Verheijen} \mathrm{M} \mathrm{J} \mathrm{and} \mathrm{Verster} \mathrm{N} \mathrm{F}$ $1981 \mathrm{~b}$ Analysis of $\mathrm{Ne}^{*}\left(1 \mathrm{~s}_{5}, 1 \mathrm{~s}_{3}\right)$ beam composition by optical pumping using non polarized light

Proc. VIII Int. Symp. on Mol. Beams, Cannes p 65

Morgenstern R, Lorents D C, Peterson J R and Olson R E 1973 Differential scattering of metastable $\mathrm{He}\left(2^{3} \mathrm{~S}\right)$ on $\mathrm{He}\left(1^{1} \mathrm{~S}\right)$ at energies between 5 and $10 \mathrm{eV}$

Phys. Rev. A8 2372

Pots B F M 1979 Turbulence and transport in a magnetized plasma PhD Thesis Eindhoven University of Technology Theuws P G A 1981 Molecular beam sampling of a hollow cathode arc PhD Thesis Eindhoven University of Technology Theuws P G A, Pernot C E E, Beijerinck H C W, Schram D C and Verster N F 1979b Production of metastables in a hollow cathode arc discharge

Proc. VII Int. Symp. on Mol. Beams, Riva del Garda p 238

Theuws P G A, Beijerinck H C W, Pernot C E E, Schram D C and Verster N F 1979a Molecular beam sampling of metastable atoms from a hollow cathode arc as a probe for the electron temperature

Proc. XIV Int. Conf. on the Phys. of Ion. Gases (Coll. no. 7, Suppl.J. Physique) Grenoble p 141

Theuws P G A, Beijerinck H G W, Schram D C and Verster N F 1977a Molecular beam sampling of a hollow cathode discharge in argon as a plasma diagnostic and a source for fast neutrals

J.Appl. Phys. 482261

Theuws P G A, Beijerinck H C W, Uitterhoeve P J, Schram D $\mathrm{C}$ and Verster N F $1977 \mathrm{~b}$ A hollow cathode discharge as a high intensity source for fast neutrals in the $\mathrm{eV}$ range

Proc. VI Int. Symp. on Mol. Beams, Noordwijkerhout p 265

Theuws P G A, Beijerinck H C W and Verster N F 1982

Detection of ground state atoms by electron excitation to a metastable level

J. Phys. E: Sci. Instrum. 15 328-33

Timmermans C J, Lunk A and Schram D C 1981 The rotation of ions and neutrals in a cylindrical magnetized hollow cathode argon arc

Beitr. Plasmaphys. 21117

Van der Kam P M A 1981 Absolute total cross sections for noble gas systems

PhD Thesis Eindhoven University of Technology

Van der Kam P M A, Beijerinck H C W and Verster N F 1981 Glory undulations in the total cross section for $\mathrm{Ar}-\mathrm{Ar}, \mathrm{Kr}, \mathrm{Xe}$ and $\mathrm{Kr}-\mathrm{Ar}, \mathrm{Kr}$ submitted for publication.

Van der Sijde B 1972 Configuration temperatures in a hollow cathode argon are and transition probabilities of the argon II spectrum

J. Quant. Spectrosc. Radiat. Transfer 12703

Way K R, Yang S C and Stwalley W C 1976 Arc-heated highintensity source of hydrogen atoms

Rev. Sci. Instrum. 471049

Young W S, Rodgers W E and Knuth E L 1969 An arc heater for supersonic molecular beams

Rev. Sci. Instrum. 401346 